\title{
48, XXYY Genotype: Case Report of a Rare Clinical Syndrome
}

\author{
Arsha Sreedhar ${ }^{1 *}$, Deborah Feden ${ }^{1}$, Gretchen Perilli ${ }^{2}$, Ranjit Nair ${ }^{3}$, VasudevMagaji ${ }^{2}$ \\ ${ }^{1}$ Department of Internal Medicine, Lehigh Valley Health Network, Allentown, PA 18101, USA \\ ${ }^{2}$ Department of Endocrinology, Lehigh Valley Health Network, Allentown, PA 18103, USA \\ ${ }^{3}$ Department of Hematology and Oncology, Lehigh Valley Health Network, Allentown, PA 18103, USA
}

Received: June 11, 2015; Accepted: December 17, 2015; Published: January 01, 2016

*Corresponding author: Arsha Sreedhar, Department of Internal Medicine, Lehigh Valley Health Network, One City Center, 707 Hamilton Street, 9 th Floor Allentown, PA 18101, USA, Tel: +484-862-3159; Fax: +484-862-3175; E-mail: arshasreedhar@gmail.com

\begin{abstract}
48, XXYY syndrome is extremely rare with an incidence of 1:18000-1: 100,000 males. Classic Klinefelter Syndrome (KS) with 47, XXY karyotype has well recognized clinical features which includes seminiferous tubule dysgenesis, androgen deficiency, cognitive and behavioral dysfunction, increased risk of autoimmune disorders, thromboembolic disease and cancer. However the clinical features of 48 , XXYY syndrome are extremely diverse and more pronounced in comparison to classic KS. With each additional X chromosome there is progressive divergence from the normal with increased frequency of phenotypic, endocrine and structural abnormalities. We describe here the case of a 28-year old male patient, who was diagnosed with classic KS at a very young age of 14, presented with not only behavioral and cognitive impairment but also with a wide spectrum of medical complications. His distinct clinical features led a comprehensive re-evaluation with repeat karyotyping and was identified as XXYY genotype.
\end{abstract}

\section{Introduction}

Klinefelter Syndrome (KS) is the most common sex chromosomal disorder which occurs in approximately 1 per 500 live born male births [1]. The characteristic clinical features of KS with the classic 47, XXY karyotype is well recognized which includes seminiferous tubule dysgenesis, androgen deficiency, cognitive and behavioral dysfunction, increased risk of autoimmune disorders, thromboembolic disease and cancer [2]. With each additional $\mathrm{X}$ chromosome there is progressive divergence from the normal with increased frequency of phenotypic, endocrine and structural abnormalities [3]. Poly $\mathrm{X}$-syndrome, often described in literature as a variant of $\mathrm{KS}$, is much rarely reported with a prevalence of 1:18,000-1:100,000 male births and has more pronounced congenital malformations and associated medical comorbidities [4]. We describe a case of a patient who had a formal diagnosis of classic KS from a very young age of 14 , presented with not only behavioral and cognitive impairment but also with a wide spectrum of medical complications and further identified on reassessment as XXYY genotype.

\section{Case}

A 28-year-old male with a known diagnosis of KS presented to our office for a routine visit. The diagnosis of classic KS was initially made during a learning disability evaluation at the age of 14. Intriguingly his medical history was very complicated for his very young age.

At age 13 he was diagnosed with common variable immunodeficiency and had recurrent upper respiratory infections until the initiation of immunoglobulin therapy. He suffered from childhood asthma with severe restrictive ventilatory defect on pulmonary function test. His other co-morbidities at that time included type 2 diabetes, metabolic syndrome with evidence of moderate sleep apnea. He had history of very low testosterone level with previous testosterone replacement however with he has been extremely non-compliant to treatment. He suffered NSTEMI at the age of 15 for which he has been on aspirin. His recent echocardiogram showed preserved ejection fraction, severe left ventricular hypertrophy and hypokinesis of the basal inferoseptum consistent with his history of myocardial infarction. By the age of 16, he developed 3 episodes of unprovoked Deep Vein Thrombosis (DVT) and further an episode of acute embolic cerebrovascular accident. Subsequent evaluation with transesophageal echocardiogram showed septum Secundum Atrial Septal Defect (ASD) with Patent Foramen Ovale (PFO) which contributed to the paradoxical embolic event. Due to multiple cardiovascular events he underwent surgical repair of PFO. His multiple DVTs despite anticoagulation prompted IVC filter insertion.

He has significant psychiatric history including bipolar disorder with multiple hospitalizations for agitation, impulsive behavior, auditory hallucinations and suicidal ideations. His diagnosis included oppositional defiant disorder, learning disability and mild mental retardation with an IQ of 62. The patient was on maintenance dose of lithium which required discontinuation at a later period secondary to an episode of renal failure. Subsequently he has been on high dose of quetiapine and citalopram. He continued to have outbursts and periods of agitation lasting for minutes but was easily redirectable.

His current physical examination revealed a tall stature with a height 76.8 inch and weighed 166 kilogram. He had sparse 
facial and body hair, female fat distribution, firm atrophic testis and bilateral gynecomastia. CT imaging studies of head showed underdeveloped left frontal sinus with poor dentition (Figure 1). Cervical spine imaging showed loss of cervical lordosis (Figure 2). Plain radiography showed congenital dysplastic anomaly of right radial head (Figure 3).

Due to his multiple diverse medical problems, a karyotypic analysis was repeated at our office which revealed $48, \mathrm{XXYY}$ genotype.

\section{Discussion}

KS is named after Harry Klinefelter, who first described the syndrome in 1942 [5,6]. In 1959, Jacobs, et al. [7] described XXY as the chromosomal constitution of KS. KS results from extra $\mathrm{X}$ chromosome in $80 \%$ of cases while in the remaining cases

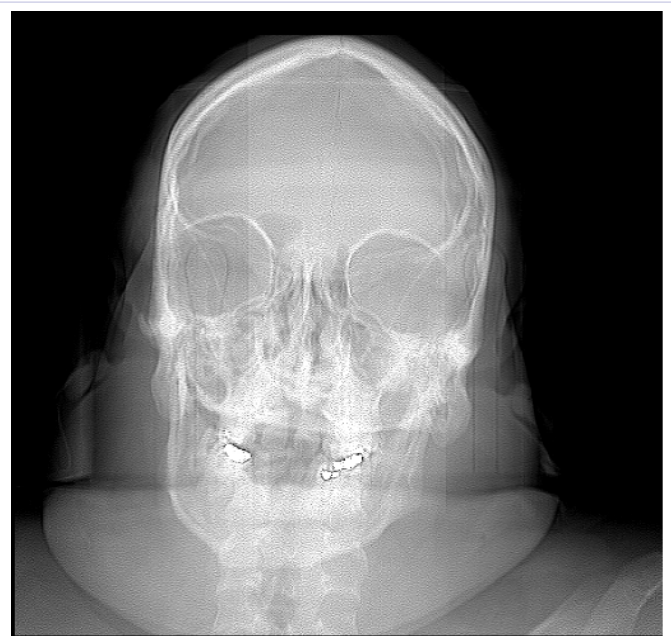

Figure 1: Craniofacial abnormalities-CT image showing underdeveloped left frontal sinus with poor dentition.

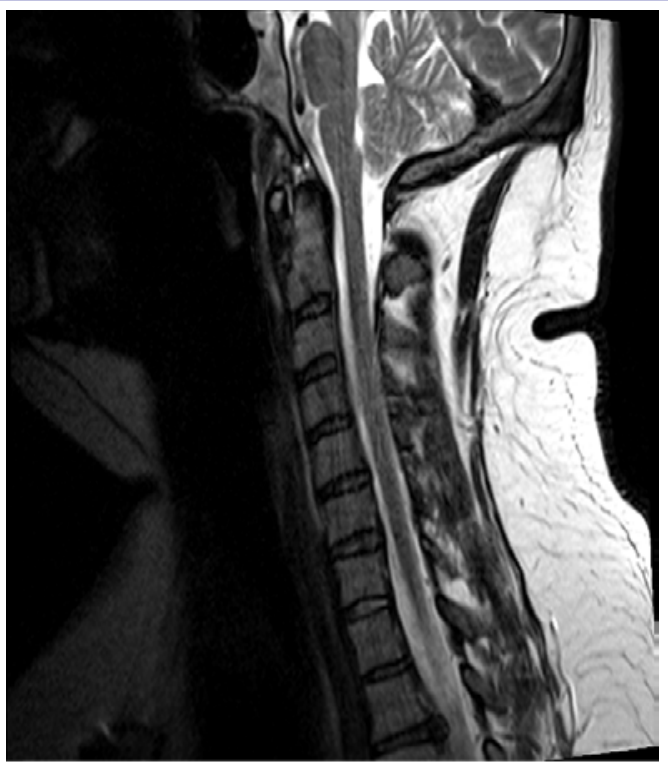

Figure 2: Cervical spine imaging showed loss of cervical lordosis.

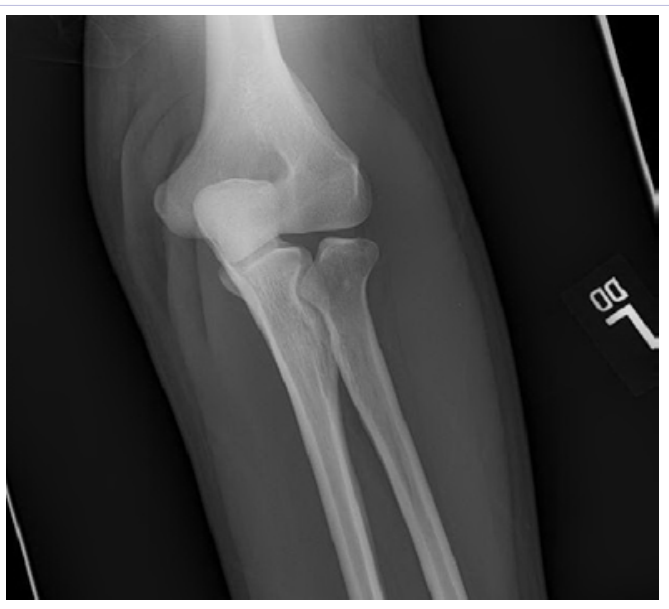

Figure 3: Congenital deformity of head of radius..

this arises from numerous sex chromosome abnormalities (48, XXXY, 48, XXYY, 49, XXXXY) or 46, XY/ 47, XXY mosaicism, or structurally abnormal sex chromosomes [8]

Due to distinguishable symptoms, physical features and multiple long term complications 48, XXYY syndrome should be considered a distinct clinical syndrome. 48, XXYY syndrome is extremely rare with an incidence of 1:18000 - 1: 100,000 males [4,9-11]. 48, XXYY syndrome is usually caused by double non-disjunction occurring at both the first and second meiotic division during spermatogenesis and fusion of the resulting 25, $\mathrm{XYY}$ spermatozoon with a 23, X ovum, or nondisjunction at the second meiotic division in both parents resulting in the fusion between a 24, XX ovum and a 24, YY sperm [2]. It has been studied that in poly-X KS: 48, XXXY and 49, XXXXY, that the additional sex chromosomes almost always arise from a single parent, usually the mother while in $48, \mathrm{XXYY}$ it is paternal in origin [4] .

The physical features of 48 , XXYY syndrome are similar to 47 , XXY but with more pronounced phenotypic abnormalities including craniofacial dysmorphism, skeletal anomalies such as radioulnar, synostosis and clinodactyly, lower cognitive function resulting in developmental delays, congenital cardiac anomalies, hypogonadism and asthma manifesting at younger age [1,9]. 48, XXYY patients are often tall with an eunuchoid habitus with adult height above 6 feet $[1,9]$. Other common clinical features include sparse body hair, small testicles and penis and gynecomastia. Peripheral vascular disease may result in leg ulcers and varicosities. There is a reported higher rate of hospitalizations among Poly $\mathrm{X}$ syndrome patients for respiratory infections and asthma exacerbations pointing towards immunological dysfunctions and subsequent infections [9].

There is increased reported incidence of congenital heart disease reported in 48, XXYY syndrome which includes septal defect, tetralogy of fallot, pulmonary stenosis, mitral valve prolapsed [9]. Our patient had septum secundum ASD which accounted for the paradoxical cerebrovascular accident.

Autism spectrum disorders have been reported in more than $1 / 3$ rd and ADHD in $70 \%$ of 48 , XXYY syndrome $[4,12]$. 
Their behavior abnormalities include hyperactivity, attention problems, impulsivity, aggression, mood instability, autistic-like behaviors, and poor social function [9]. In classic KS, the typical cognitive pattern shows deficit in specific domains of cognition including language and frontal-executive functions, rather than a general decline in intellectual ability [13]. 48, XXYY males have significantly lower IQ and language disability than classic 47, XXY with studies showing an IQ reduction of 15-16 points with every additional X chromosome $[1,14]$. They are usually shy however can be aggressive and impulsive with significant academic, social and behavioral deficits [1]. 48, XXYY males have significantly lower verbal IQ (VIQ) when compared to Performance IQ (PIQ) and interestingly the VIQ decreases with age while PIQ remains mostly unchanged [12]. As a result 48, XXYY males have significantly lower language associated disability and adaptive functioning than males with classic 47, XXY [1,4].

Our patient had DVT at a very early age of 16 with multiple recurrences. There is increased incidence of DVT in 48, XXYY syndrome affecting more than $20 \%$ over the age of 20 years. The pathophysiology this not been elucidated but could be attributed to estrogen testosterone imbalance [4]. Type II diabetes is seen in about $18 \%$ of patients with KS 48 , XXYY. Hypergonadotropic hypogonadism is common in pubertal-aged boys which would require treatment with testosterone replacement therapy [9]. Behavioral issues in these groups of patients can make the management of these extremely challenging. The association of classic KS, 47, XXY with malignant diseases is well known. An analysis of cancer incidence by karyotype revealed that men with 48, XXYY had a particularly elevated incidence of non-Hodgkin lymphoma, but due to the rarity of this syndrome there is no conclusive data on the incidence of specific malignancies in 48 , XXYY [15].

Medical work up at the time of diagnosis should include a baseline evaluation including comprehensive neurodevelopmental assessment, a cardiac evaluation for congenital heart defects, renal ultrasound due to association with unilateral kidney and orthopedic evaluation for skeletal abnormalities. Other test should be considered based on clinical symptoms. Adolescents and adults should also be screened for diabetes, hypothyroidism, hypogonadism, osteoporosis, and obesity. All patients need to be screened for autism spectrum disorders, ADHD, mood disorders and other mental health problems. We underscore the importance of having a high index of suspicion for deep vein thrombosis, acute coronary syndrome and non-Hodgkin lymphoma based on clinical symptoms [9]. Management should include a multidisciplinary approach including experts in endocrinology, psychiatry, neurology, speech/language therapy, occupational therapy, and physical therapy.

\section{Conclusion}

This case presents important comparisons between the phenotypes of 48, XXYY syndrome and the classic 47, XXY karyotype. Early recognition, thorough neurodevelopmental assessment and close long term follow up is critical in patients with 48, XXYY syndrome due to its multiple medical, psychosocial and life threatening associations.

\section{References}

1. Visootsak J, Graham Jr JM. Klinefelter syndrome and other sex chromosomal aneuploidies. Orphanet J Rare Dis. 2006; 1(42): 1-5.

2. Bischoff FZ, Elias S. Klinefelter Syndrome. The Global Library of Women's Medicine. (ISSN: 1756-2228) 2008; DOI: 10.3843/ GLOWM.10361.

3. Simpson JL, de la Cruz F, Swerdloff RS, Samango-Sprouse C, Skakkebaek NE, Graham JM, et al. Klinefelter syndrome: expanding the phenotype and identifying new research directions. Genetics in Medicine. 2003; 5(6): 460-468.

4. Tartaglia N, Ayari N, Howell S, D’Epagnier C, Zeitler P. 48, XXYY, 48, XXXY and 49, XXXXY syndromes: not just variants of Klinefelter syndrome. Acta Paediatr. 2011; 100 (6): 851-860. doi: 10.1111/j.16512227.2011.02235.x

5. Klinefelter HF. Klinefelter's syndrome: historical background and development. South Med J. 1986; 79(9): 1089-93.

6. Klinefelter Jr HF, Reifenstein Jr EC, JR FULLER A JR. Syndrome characterized by gynecomastia, aspermatogenesis without A-Leydigism, and increased excretion of follicle-stimulating hormone. The Journal of Clinical Endocrinology \& Metabolism. 1942; 2(11): 615-627.

7. Jacobs, Patricia A. A case of human intersexuality having a possible XXY sex-determining mechanism. Nature. 1959; 183(4657): 302-303.

8. Frühmesser A, Kotzot D. Chromosomal variants in Klinefelter syndrome. Sexual Development. 2011; 5(3): 109-123. doi: 10.1159/000327324.

9. Tartaglia N, Davis S, Hench A, Nimishakavi S, Beauregard R, Reynolds A, et al. A new look at XXYY syndrome: medical and psychological features. American Journal of Medical Genetics Part A. 2008; 146(12): 1509-1522. doi: 10.1002/ajmg.a.32366.

10.Zelante L, Piemontese MR, Francioli G, Calvano S. Two 48, XXYY patients: clinical, cytogenetic and molecular aspects. Ann Genet. 2003; 46(4): 479-481.

11.Visootsak J, Rosner B, Dykens E, Tartaglia N, Graham JM Jr. Behavioral phenotype of sex chromosome aneuploidies: 48, XXYY, 48, XXXY, and 49, XXXXY. Am J Med Genet A. 2007; 143(11): 1198-1203.

12. Cordeiro L, Tartaglia N, Roeltgen D, Ross J. Social deficits in male children and adolescents with sex chromosome aneuploidy: a comparison of XXY, XYY, and XXYY syndromes. Res Dev Disabil. 2012; 33(4): 1254-1263. doi: 10.1016/j.ridd.2012.02.013.

13. Messina MF, Sgrò DL, Aversa T, Pecoraro M, Valenzise M, De Luca F. A characteristic cognitive and behavioral pattern as a clue to suspect Klinefelter syndrome in prepubertal age. J Am Board Fam Med. 2012; 25(5): 745-749. doi: 10.3122/jabfm.2012.05.110232.

14.Dada R, Kumar R, Ahmed ME. Klinefelter Syndrome: Clinical implications of expanding phenotype. Indian J Practising Doctor. $2007 ; 3(6): 1-2$.

15. Swerdlow AJ, Schoemaker MJ, Higgins CD, Wright AF, Jacobs PA; UK Clinical Cytogenetics Group. Cancer incidence and mortality in men with Klinefelter syndrome: a cohort study. J Natl Cancer Inst; 97(16): 1204-1210. 